\title{
Hydrodynamical description for magneto-transport in the strange metal phase of Bi-2201
}

\author{
Andrea Amoretti $\odot,{ }^{1,2}$ Martina Meinero, ${ }^{1,3}$ Daniel K. Brattan, ${ }^{2}$ Federico Caglieris, ${ }^{4}$ Enrico Giannini $\odot,{ }^{5}$ Marco Affronte, ${ }^{6}$ \\ Christian Hess, ${ }^{4,7}$ Bernd Buechner, ${ }^{4,7}$ Nicodemo Magnoli $\odot,{ }^{1,2}$ and Marina Putti ${ }^{1,3}$ \\ ${ }^{1}$ Dipartimento di Fisica, Università di Genova, via Dodecaneso 33, I-16146, Genova, Italy \\ ${ }^{2}$ I.N.F.N. - Sezione di Genova, via Dodecaneso 33, I-16146, Genova, Italy \\ ${ }^{3}$ CNR-SPIN, Corso Perrone 24, 16152 Genova, Italy \\ ${ }^{4}$ Leibniz IFW Dresden, Helmholtz str. 20, D-01069 Dresden, Germany \\ ${ }^{5}$ Department of Quantum Matter Physics, University of Geneva, 24 Quai Ernest Ansermet, CH-1211 Geneva, Switzerland \\ ${ }^{6}$ CNR Nano Istituto Nanoscience - sezione S3 and Universitá di Modena e Reggio Emilia - Dipartimento di Scienze Fisiche, \\ Informatiche e Matematiche, via G. campi 213/A, 41125 Modena, Italy \\ ${ }^{7}$ Faculty of Physics, Technische Universität Dresden, D-01062 Dresden, Germany
}

(Received 24 October 2019; revised manuscript received 31 March 2020; accepted 8 June 2020; published 23 June 2020)

\begin{abstract}
High-temperature superconductors are strongly coupled systems which present a complicated phase diagram with many coexisting phases. This makes it difficult to understand the mechanism which generates their singular transport properties. Hydrodynamics, which mostly relies on the symmetries of the system without referring to any specific microscopic mechanism, constitutes a promising framework to analyze these materials. In this paper we show that, in the strange metal phase of the cuprates, a whole set of transport coefficients are described by a universal hydrodynamic framework once one accounts for the effects of quantum critical charge-density waves. We corroborate our theoretical prediction by measuring the DC transport properties of Bi-2201 close to optimal doping, proving the validity of our approach. Our argument can be used as a consistency check to understand the universality class governing the behavior of high-temperature cuprate superconductors.
\end{abstract}

DOI: 10.1103/PhysRevResearch.2.023387

\section{INTRODUCTION}

Three decades after the discovery of high- $T_{c}$ superconductors [1], the underlying mechanisms responsible for their peculiar behavior remain mostly unexplained. The superconductive region of the phase diagram changes upon doping with electrons or holes into a Mott-insulating copper oxide; the chemical doping modifies the density of carriers of the $\mathrm{CuO}_{2}$ planes, rapidly suppressing the antiferromagnetic order. Above the superconducting dome there exists a debated pseudogap phase whose origin is still enigmatic. For temperatures higher than the pseudogap temperature the appearance of a strange metallic phase, characterized by the unusual temperature dependence of the transport coefficients is one of the grand unresolved issues in these materials. A quantum critical point around the optimally doped region, whose presence would force the temperature dependence of the observables of the system to obey simple scaling laws, has been proposed to be the origin of the exotic phenomena measured in the strange metallic phase [2].

In recent years, a plethora of experiments [3-20], using a variety of distinct experimental techniques, have proven

Published by the American Physical Society under the terms of the Creative Commons Attribution 4.0 International license. Further distribution of this work must maintain attribution to the author(s) and the published article's title, journal citation, and DOI. that charge-density wave (CDW) order is an ubiquitous feature of the phase diagram of all cuprate superconductors. In particular, resonant $\mathrm{x}$-ray scattering measurements on Bi2201 [21] have shown that two-dimensional CDW order is not only present in the underdoped regime but also persists through optimal doping into the overdoped region of the phase diagram, suggesting that CDW order might play a dominant role in determining the transport properties of these materials as well.

From the theoretical point of view, the coexistence of many intertwined phases makes it difficult to develop reliable microscopic models to explain the behavior of high-temperature superconductors (see, e.g., Ref. [22] for a review). Recently, studies that try to explain the electric transport properties of these materials by relying only on the hydrodynamic universality class, without referring to any specific microscopic mechanism, have appeared [23-25].

Hydrodynamics is a tool which gives an accurate description of any interacting system, classical or quantum, as long as the fundamental interacting degrees of freedom quickly reach local thermal equilibrium. To observe hydrodynamic effects from electrons in a solid, the interaction time must be the fastest timescale in the system. In normal Fermi-liquid systems, however, the quasiparticles interact with each other very weakly, making the observation of the hydrodynamics of electron fluids notoriously hard. The situation changes in systems such as high-temperature superconductors, where the strong interactions between electrons make the quasiparticle 
Fermi-liquid picture unreliable. In these materials the only long-lived excitations are associated with (almost-)conserved quantities, like the charge current and the energy current, making hydrodynamics the ideal framework to describe the relaxation of these modes. Then, contrary to the Boltzmann equation approach, hydrodynamics does not require the existence of quasiparticles.

The hydrodynamic approach has been used successfully to analyze the properties of graphene [26] and also very recently in new strongly coupled materials [27]. In Refs. [23-25], using a hydrodynamic approach, fluctuating CDW order was proposed as an explanation for the off-axis peak in the optical conductivity developing at high temperature in many cuprates, including, in particular, BSCO and BSCCO $[28,29]$. Moreover, there is experimental evidence indicating that not only electric but also thermo-electric transport, for example, the Nernst coefficient [30], is highly affected by the presence of CDW order, not only at the fluctuating level but also closer to the superconducting phase transition.

Based on this idea, in this paper we included in the hydrodynamic theory of a two-dimensional CDW the effects of an external magnetic field $B$ perpendicular to the $\operatorname{CDW}\{x, y\}$ plane. We have analyzed how the magnetohydrodynamic approach constrains the entire set of electric, thermo-electric, and thermal DC transport coefficients. In this approach, parity is broken solely by the presence of $B$, which can be seen as an external potential. We refer to the Supplemental Material (SM) [31] for technical details. As already found in Ref. [23], the electric conductivity at low $B$ splits into two terms: an incoherent conductivity $\sigma_{0}$ which characterizes the electric transport at the quantum critical point, and an additional term $\tilde{\sigma}$ which includes the relaxation mechanisms,

$$
\sigma_{\mathrm{DC}}=\sigma_{0}+\tilde{\sigma} \text { with } \tilde{\sigma}=\frac{n^{2}}{\chi_{\pi \pi}} \frac{\Omega_{1}}{\Omega_{1} \Gamma+\omega_{0}^{2}},
$$

where $\Gamma$ is an external momentum relaxation rate which takes into account any external mechanism of momentum loss by the electronic plasma (e.g., the interactions with the underlying lattice), $n^{2} / \chi_{\pi \pi}$ is the Drude weight, and $\omega_{0}$ is the so-called pinning frequency [32-34]. The pinning frequency is a characteristic frequency for the CDW collective modes (pseudo-Goldstone bosons) and is nonzero as soon as the they acquire a small mass, e.g., in the presence of impurities or disorder. The additional parameter $\Omega_{1}$ is a phase relaxation for the collective modes associated with the CDW. Initially $\Omega_{1}$ was included to account for the consequences of topological defects [35] but, as recently noted in Refs. [36,37], it also makes a nontrivial contribution to the transport when the CDW pseudo-Goldstone bosons have a small mass, namely, in the presence of simple disorder (similarly to $\omega_{0}$ ). For $\Omega_{1}=\sigma_{0}=0$, one finds that $\sigma_{\mathrm{DC}}=0$, as already known from the standard CDW approach [32-34]. However, in the most general case with $\Omega_{1}, \sigma_{0} \neq 0$, hydrodynamics allows for a CDW with metallic behavior. Importantly, and in contrast with the standard Drude scenario, as long as $\Omega_{1}, \omega_{0} \neq 0$, the DC conductivity is finite even if $\Gamma=0$.

The other DC transport coefficients are tightly constrained in terms of a small number of parameters. Specifically, the low- $B$ behavior of the DC electric resistivity $\rho_{x x}$, the Hall angle $\cot \Theta_{H}=\sigma_{x x} / \sigma_{x y}$, the magnetoresistance $\Delta \rho / \rho$, the Nernst coefficient $N$, and the thermal Hall conductivity $\kappa_{x y}$ are of the following form:

$$
\begin{aligned}
\rho_{x x} & =\frac{1}{\sigma_{0}+\tilde{\sigma}}+O\left(B^{2}\right), \\
\frac{\Delta \rho}{\rho} & =B^{2} \frac{\sigma_{0}^{3} \tilde{\sigma}}{n^{2}} \frac{1}{\left(\sigma_{0}+\tilde{\sigma}\right)^{2}}+O\left(B^{4}\right), \\
\kappa_{x y} & =-B T \frac{\tilde{\sigma}^{2} s}{n^{4}}\left(n s+2 \frac{\alpha_{0} n^{2}}{\tilde{\sigma}}\right)+O\left(B^{3}\right), \\
\cot \Theta_{H} & =\frac{n}{B \tilde{\sigma}} \frac{1+\frac{\sigma_{0}}{\tilde{\tilde{\sigma}}}}{1+2 \frac{\sigma_{0}}{\tilde{\sigma}}}+O(B), \\
N & =\frac{B \sigma_{0} \tilde{\sigma}}{n^{2}\left(\sigma_{0}+\tilde{\sigma}\right)^{2}}\left(s \sigma_{0}-n \alpha_{0}\right)+O\left(B^{3}\right),
\end{aligned}
$$

where $\sigma_{0}$ and $\tilde{\sigma}$ are the incoherent and relaxation conductivities defined in Eq. (1), $T$ is the temperature, and $s$ and $n$ are the entropy density and the charge-carrier density, respectively. The parameter $\alpha_{0}$ is an incoherent thermo-electric conductivity. It can be expressed as a function of the chemical potential $\mu$ and $\sigma_{0}$, namely, $\alpha_{0} \sim-\mu \sigma_{0} / T$, if we assume that the low- $T$ behavior of the system is influenced by the presence of a relativistic covariant quantum critical point (see the SM [31]). This implies that the theory of quantum critical CDW hydrodynamics allows us to express the five transport coefficients in Eq. (2) as a function of only four temperature-dependent quantities: $\sigma_{0}, \tilde{\sigma}, n$, and $s$. Eventually, by measuring four of the quantities in Eq. (2), one can predict the temperature dependence of the fifth transport coefficient.

This puts hydrodynamics on a privileged ground to analyze strange metals. In fact, being agnostic on the specific microscopic mechanism responsible for the peculiar behavior of these materials, hydrodynamics provides a way to test the appropriate universality class to which these systems belong at the level of the DC transport properties.

To test the last statement, we measured the five DC magneto-transport coefficients (2) in $\mathrm{Bi}_{2} \mathrm{Sr}_{2} \mathrm{CuO}_{6}$. Being electrical and/or transverse transport coefficients, these quantities are largely independent of the effects of lattice phonons and constitute ideal observables to test the electronic properties of the material. Moreover, measuring all the transport coefficients in the same sample allows us to be confident that all the data refer to the same doping and impurity level. By fixing the temperature dependence of the four phenomenological quantities in Eq. (2) using the low-temperature behavior of $\rho_{x x}, \Delta \rho / \rho, \cot \Theta_{H}$, and $\kappa_{x y}$, we have been able to uniquely determine the low-temperature dependence of the Nernst coefficient. The obtained result is in strong agreement with our experimental observations.

\section{EXPERIMENTAL RESULTS}

Among the cuprates, $\mathrm{Bi}_{2} \mathrm{Sr}_{2} \mathrm{CuO}_{6}(\mathrm{Bi}-2201)$ is one of the most promising materials to test the magneto-hydrodynamic scenario due to its relatively low critical temperature $\left(T_{c} \approx\right.$ $10-20 \mathrm{~K})$ [38-40], which allows for a systematic study of 

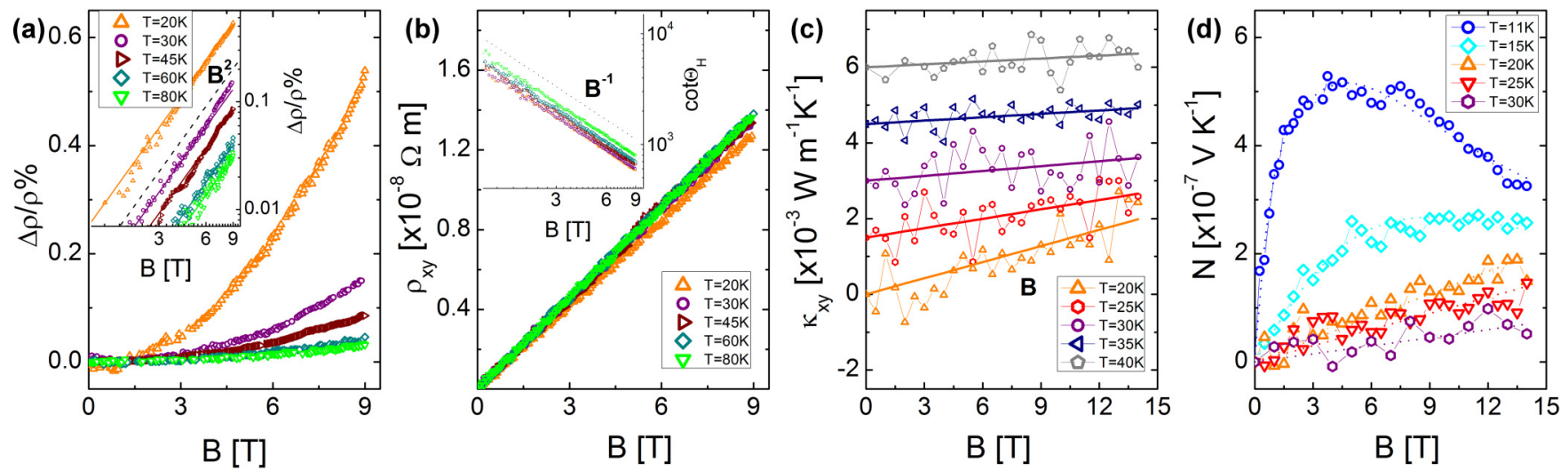

FIG. 1. Magnetic-field dependence of (a) $\Delta \rho / \rho$, (b) $\rho_{x y}$ and ([inset of panel (b)] cot $\Theta_{H}$, (c) $\kappa_{x y}$ together with its linear fits (solid lines), and (d) $N$ (dashed lines are to guide the eye). In panel (c), the curves are shifted to avoid overlapping.

its normal-state properties down to low temperatures. ${ }^{1}$ In this compound, the main signatures of quantum criticality have been reported: its electric resistivity is $T$-linear up to $700 \mathrm{~K}$ [42] and CDW modulations have been observed in a wide region of its phase diagram [21].

We performed our measurements on single crystals of Bi2201 close to optimal doping. The electric characterization has been undertaken with several samples and we report here the data relative to three crystals, named $\mathrm{L} 2, \mathrm{~K} 8$, and $\mathrm{K} 10$, whereas the thermal and thermoelectric characterizations have been performed on the L2 sample (its size being suitable for these measurements). The in-plane resistivity $\rho_{x x}$ of the L2, $\mathrm{K} 8$, and $\mathrm{K} 10$ samples as a function of temperature shows a quite sharp superconducting transition at $T_{c} \approx 11 \mathrm{~K}$ and, above this temperature, $\rho_{x x}(T)$ is almost linear up to $380 \mathrm{~K}$ (see the SM for details [31]).

Figure 1(a) displays the magnetoresistance, defined as $\Delta \rho / \rho=\left[\rho_{x x}(B)-\rho_{x x}(0)\right] / \rho_{x x}(0)$, of the L2 sample as a function of $B$ up to $9 \mathrm{~T}$ applied in the out-of-plane direction, for selected temperatures in the range $20-80 \mathrm{~K}$. As evinced by the bi-logarithmic scale reported in the inset, $\Delta \rho / \rho$ increases as $B^{2}$, as expected for the orbital contribution. The values are quite small (lower than $0.5 \%$ ) and they abruptly decrease with temperature.

In Fig. 1(b) we report the transverse resistivity $\rho_{x y}$ of the L2 sample as a function of $B$ up to $9 \mathrm{~T}$ applied in the out-of-plane direction, at selected temperatures in the range from 20 to $80 \mathrm{~K} . \rho_{x y}$ is $B$ linear and it exhibits a weak temperature dependence. The inset of Fig. 1(b) shows the bilogarithmic plot of the inverse Hall angle $\cot \Theta_{H}=\rho_{x x} / \rho_{x y}$, which is proportional to $B^{-1}$ as a consequence of the small magnetoresistance and the linear dependence of $\rho_{x y}$. The same behavior has been measured also for K7 and K10 samples.

Figure 1(c) shows the magnetic-field dependence up to $14 \mathrm{~T}$ of the thermal Hall conductivity $\kappa_{x y}$ measured at selected temperatures in the range $20-40 \mathrm{~K}$ (see the SM). It grows linearly with $B$, reaching values lower than $2 \times 10^{-3} \mathrm{~W}$ $\mathrm{m}^{-1} \mathrm{~K}^{-1}$ measured at $20 \mathrm{~K}$ and $14 \mathrm{~T}$ (solid lines are linear

\footnotetext{
${ }^{1}$ It has been reported that similar compound might have a complicated Fermi surface, presenting pockets [41], even though the relevance of these pockets on the transport properties remains unclear.
}

fits to the data and the curves are shifted to avoid overlapping) with a pretty small slope, which monotonically decreases with increasing temperature. Above $40 \mathrm{~K}, \kappa_{x y}$ is vanishingly small, so that no meaningful signal can be extracted from the noise.

In Fig. 1(d) we report the magnetic-field dependence of $N$ from 0 to $14 \mathrm{~T}$ at $T=11,15,20,25$, and $30 \mathrm{~K}$. At $11 \mathrm{~K}$, just above $T_{c}, N$ shows the characteristic "tilted-hill" profile which has been widely ascribed to contributions from superconducting vortices [43]. At $T=15 \mathrm{~K}$, the vortex contribution is still visible, even if markedly reduced. For $T \geqslant 20 \mathrm{~K}$, $N$ is $B$-linear with a slope which slowly decreases with increasing $T$. We therefore exclude any vortex contribution above $20 \mathrm{~K}$ where we still observe a non-negligible Nernst signal, already reported in cuprates, whose origin remains controversial $[43,44]$.

The magnetohydrodynamic expansion for a parityinvariant system gives that the transport coefficients behave as reported in Eqs. (2), namely, $\Delta \rho \sim B^{2}, \cot \Theta_{H} \sim B^{-1}$, $\kappa_{x y} \sim B$, and $N \sim B$ (see SM [31]). As shown in Fig. 1, these predictions agree with the experimental results above $20 \mathrm{~K}$ since, below this temperature, the Nernst effect is influenced by vortex contributions.

In addition to the magnetic-field scalings, we have analyzed the temperature dependence of $\Delta \rho / \rho, \cot \Theta_{H}, k_{x y}$, and $N$. Figure 2(a) displays the bi-logarithmic plot of $\Delta \rho / \rho$ at $B=9 \mathrm{~T}$ for selected temperatures in the range $10-100 \mathrm{~K}$. We note that the data relative to the $\mathrm{L} 2, \mathrm{~K} 10$, and $\mathrm{K} 7$ samples almost overlap between 15 and $40 \mathrm{~K}$, where $\Delta \rho / \rho$ decreases by about two orders of magnitude, as fast as $\sim T^{-4}$. Above $40 \mathrm{~K}, \Delta \rho / \rho$ is extremely small and the data relative to the three samples are much scattered so that it is hard to draw any conclusion about the temperature dependence for $T>40 \mathrm{~K}$.

Figure 2(b) shows the bi-logarithmic plot of $\cot \Theta_{H}=$ $\rho_{x x} / \rho_{x y}$ at $B=9 \mathrm{~T}$ for selected temperatures in the range $15-150 \mathrm{~K}$ of the $\mathrm{L} 2, \mathrm{~K} 10$, and $\mathrm{K} 7$ samples. This plot provides evidence for the good reproducibility of the data, which fall on a line $T^{1.5}$

Figure 2(c) shows the bi-logarithmic plot of $k_{x y}$ between 20 and $40 \mathrm{~K}$ at $B=14 \mathrm{~T}$. It decreases by rising $T$ as fast as $T^{-3}$.

The inset of Fig. 2(d) shows the temperature dependence of the Nernst coefficient $N$ at $B=14 \mathrm{~T}$, in the range $15-200 \mathrm{~K}$. $N$ is small and negative at high temperatures, whereas it starts to be enhanced towards positive values for $T<T_{v} \approx 130 \mathrm{~K}$, 

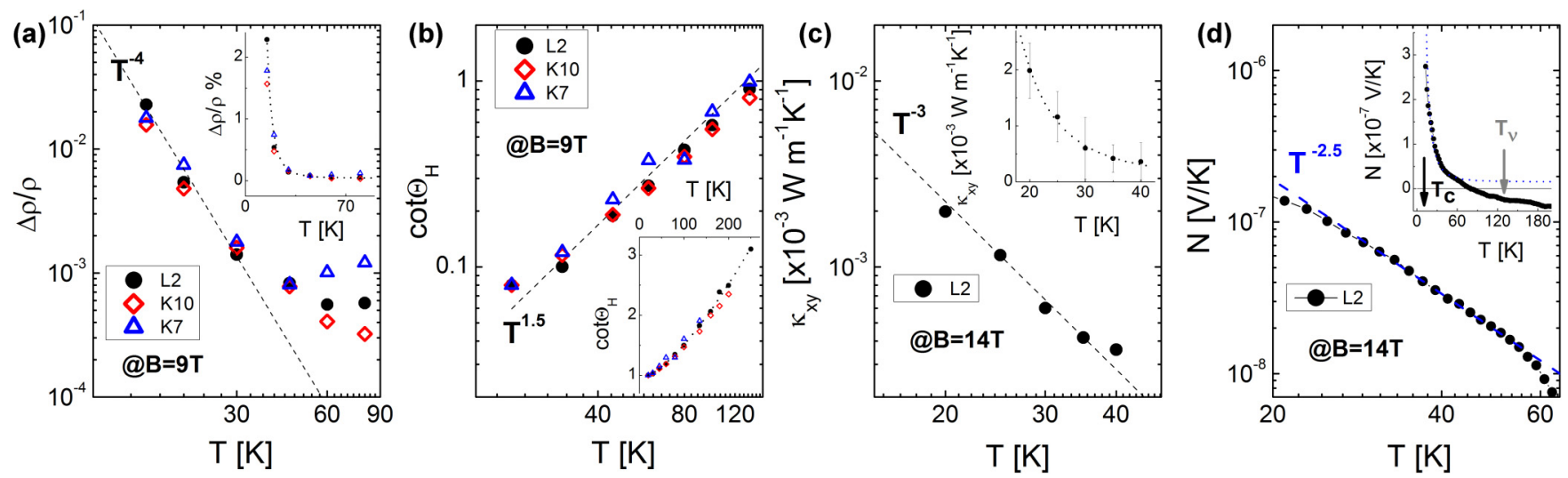

FIG. 2. Bi-logarithmic plot of (a) $\Delta \rho / \rho$ vs $T$, (b) $\cot \Theta_{H}$ vs $T$, (c) $\kappa_{x y}$ vs $T$, and (d) $N$ vs $T$, together with functions which well reproduce the data (dashed lines) for L2, K7, and K10 samples. Linear plots of the data are reported in the insets.

$T_{v}$ [marked with a gray arrow in inset of Fig. 2(d)] being the onset of the upturn in $N$ (see Methods for the estimation of $T_{v}$ ).

Figure 2(d) shows the bi-logarithmic plot of $N$ in the temperature range $20-60 \mathrm{~K}$ (black dots) together with the function $T^{-2.5}$ which reproduces the temperature dependence of the data above $20 \mathrm{~K}$ and below $60 \mathrm{~K}$. Indeed, as discussed above, below $20 \mathrm{~K}$ the vortex contribution prevails, whereas above $60 \mathrm{~K}, N$ changes sign (see the inset), indicating that another mechanism comes into play.

In summary, in the temperature range $20 \mathrm{~K}<T<60 \mathrm{~K}$ where the system is far enough from the superconducting transition and the thermal and thermoelectric observables are significantly different from zero, the temperature behaviors for the measured properties are the following: $\rho_{x x} \sim T$, $\Delta \rho / \rho \sim T^{-4}, \cot \Theta_{H} \sim T^{1.5}, \kappa_{x y} \sim T^{-3},{ }^{2}$ and $N \sim T^{-2.5}$.

\section{MATCHING EXPERIMENT WITH HYDRODYNAMIC PREDICTIONS}

The only consistent way to fit the behavior of the resistivity $\rho_{x x}$ [Eq. (2a)] and the Hall angle $\cot \Theta_{H}$ [Eq. (2d)] with the hydrodynamic prediction is that the quantum critical incoherent conductivity $\sigma_{0}$ is dominating $\rho_{x x}$ at low $T$. On the other hand, $\cot \Theta_{H}$ is uniquely determined by the ratio $n / \tilde{\sigma}$, which can be fixed separately. Eventually, $\rho_{x x} \sim \frac{1}{\sigma_{0}} \sim T$, $\cot \Theta_{H} \sim \frac{n}{B \tilde{\sigma}} \sim T^{1.5}$. This is a known hydrodynamic mechanism [45-47]. In contrast with the Fermi liquid, the fact that the electric conductivity splits into two terms $\left(\sigma_{D C}=\right.$ $\sigma_{0}+\tilde{\sigma}$ ) allows for two different mechanisms to determine the behavior of the resistivity and the Hall angle. In this case, the incoherent quantum critical conductivity dominates the electric longitudinal transport while the relaxation conductivity $\tilde{\sigma}$ determines the temperature dependence of the Hall angle.

Imposing the temperature behavior of the magnetoresistance in Eq. (2b), $\Delta \rho / \rho \sim T^{-4}$, fixes the scalings of $n \sim$

\footnotetext{
${ }^{2}$ As already mentioned, it was not possible to separate $\kappa_{x y}$ from the noise above $40 \mathrm{~K}$ due to the vanishingly small value of the signal. However, we tentatively extend up to $60 \mathrm{~K}$ the range of validity of $\kappa_{x y} \sim T^{-3}$ to test if a consistent scenario including all the transport coefficients can be drawn.
}

$T^{1.5}$ and $\tilde{\sigma} \sim T^{0}$ separately. The charge-carrier density $n$ is decreasing with $T$, making the incoherent part of the conductivity $\sigma_{0}$ dominant at low temperatures.

To fix the temperature dependence of the entropy density $s$ we use $\kappa_{x y} \sim T^{-3}$ [Eq. (2c)]. In particular,

$$
\kappa_{x y} \sim \mu B \frac{\sigma_{0} \tilde{\sigma}}{n^{2}} s \sim T^{-3} \Rightarrow s \sim T,
$$

where we have imposed that $\alpha_{0}=-\mu \sigma_{0} / T$ close to the quantum critical point. In the SM [31] we report specific-heat measurements of the BSCO crystal, which confirm the linear dependence with temperature of the electronic specific heat, in accordance with Eq. (3). Remarkably, an entropy linear in temperature is in accordance with the early measurements of the specific-heat on optimally doped YBCO performed by Loram et al. [48].

Having fixed all the four quantities appearing in Eq. (2), the temperature dependence of the Nernst coefficient is now fully determined: Specifically,

$$
N \sim \frac{\mu B \tilde{\sigma}}{n T} \sim \frac{\mu}{T \cot \Theta_{H}} \sim T^{-2.5} .
$$

Remarkably, Eq. (4) perfectly agrees with the experimentally observed temperature dependence of $N$ between 20 and $60 \mathrm{~K}$ [Fig. 2(d)]. Analogously to the Hall angle, $\tilde{\sigma}$ completely determines the behavior of $N$. It is important to note that in Ref. [30] the enhancement of $N$ below $T_{v}$ has been interpreted as a signal of Fermi-surface reconstruction due to the onset of charge order, which is measured to set in at $\approx T_{\nu} / 2$. For our system this would imply that CDW order is the dominant mechanism for $T<65 \mathrm{~K}$, suggesting that the CDW parameters $\Omega_{1}$ and $\omega_{0}$ should dominate over $\Gamma$, so that $\tilde{\sigma}=\frac{n^{2}}{\chi_{\pi \pi}} \frac{\Omega_{1}}{\omega_{0}^{2}}$. To fulfill the scaling previously determined $\Omega_{1} / \omega_{0}^{2} \sim T^{-3}$, which can be achieved if $\Omega_{1} \sim T, \omega_{0} \sim T^{2}$. In a scale-invariant theory governed only by a relativistic quantum critical point one would expect $\Omega_{1} \sim \omega_{0} \sim T$; here, however, $\omega_{0}$ is proportional to the mass of the CDW pseudoGoldstone boson (see SM [31]), which defines an additional scale allowing for $\Omega_{1} \sim T, \omega_{0} \sim T^{2}$. In the opposite scenario with $\Gamma$ being the dominant scale, one find $\tilde{\sigma}=\frac{n^{2}}{\chi_{\pi \pi} \Gamma}$, implying $\Gamma \sim T^{3}$. This would be compatible in a clean and multiband 
metal with electron-phonon scattering being the dominant mechanism, which is not our case.

\section{OUTLOOK}

This paper provides a consistency check, based on the analysis of DC magneto-transport coefficients, of hydrodynamics being the correct framework to describe the transport properties of the strange metal phase of cuprates. Hydrodynamics constrains the whole set of transport coefficients in a tight way and implies that both the thermodynamic properties and the relaxation rates of the electronic system must have a very specific behavior in order to fit the model. Two main future directions are at hand: First, even though evidence has been reported to suggest that $\tilde{\sigma}$ is dominated by the CDW, comparing optical measurements of the electric conductivity against hydrodynamic predictions will allow us to make a conclusive statement.

Second, it is worth mentioning that, for other systems and doping levels, different scaling behavior for the transport coefficients are reported. $\cot \Theta_{H}$ has been observed to scale as $T^{2}$ in other cuprates [49,50], and different temperature dependencies of $\kappa_{x y}$ have been reported for overdoped $\mathrm{Nd}-/$ Eu-LSCO [51] and optimally doped YBCO [52,53]. Also, the electronic specific heat of YBCO has been observed to be doping dependent [48]. The measurements of the complete set of transport properties in these and other materials, performed again on the same sample, will allow us to better test the range of validity of the hydrodynamic approach and to make a firm statement on the mechanism governing the transport properties of cuprates in different regions of the phase diagram.

\section{ACKNOWLEDGMENTS}

We acknowledge discussions with I. Pallecchi and G. Lamura. A.A. would like to thank Daniel Arean, Blaise Gouteraux, Daniele Musso, and Paolo Solinas for countless discussions on related topics. The work of M.M. has been partially supported by a DAAD Scholarship, Research Grants Award - Short-term grants, 2017 (57314023).
[1] G. Bednorz and K. A. Müller, Possible high- $T_{c}$ superconductivity in the Ba-La-Cu-O system, Z. Phys. B: Condens. Matter 64, 189 (1986).

[2] Subir Sachdev and Jinwu Ye, Universal Quantum-Critical Dynamics of Two-Dimensional Antiferromagnets, Phys. Rev. Lett. 69, 2411 (1992).

[3] J. M. Tranquada, B. J. Sternlieb, J. D. Axe, Y. Nakamura, S. Uchida, Evidence for stripe correlations of spins and holes in copper oxide superconductors, Nature (London) 375, 561 (1995).

[4] M. Fujita, H. Goka, K. Yamada, and M. Matsuda, Competition Between Charge and Spin-Density-Wave Order and Superconductivity in $\mathrm{La}_{1.875} \mathrm{Ba}_{0.125-x} \mathrm{Sr}_{x} \mathrm{CuO}_{4}$, Phys. Rev. Lett. 88, 167008 (2002).

[5] P. Abbamonte et al., Spatially modulated "Mottness" in $\mathrm{La}_{2-x} \mathrm{Ba}_{x} \mathrm{CuO}_{4}$, Nat. Phys. 1, 155 (2005).

[6] J. E. Hoffman et al., A four unit cell periodic pattern of quasiparticle states surrounding vortex cores in $\mathrm{Bi}_{2} \mathrm{Sr}_{2} \mathrm{CaCu}_{2} \mathrm{O}_{8+\delta}$, Science 295, 466 (2002).

[7] T. Wu et al., Magnetic-field-induced charge-stripe order in the high-temperature superconductor $\mathrm{YBa}_{2} \mathrm{Cu}_{3} \mathrm{O}_{y}$, Nature (London) 477, 191 (2011).

[8] G. Ghiringhelli et al., Long-range incommensurate charge fluctuations in (Y, Nd) $\mathrm{Ba}_{2} \mathrm{Cu}_{3} \mathrm{O}_{6+x}$, Science 337, 821 (2012).

[9] J. Chang et al., Direct observation of competition between superconductivity and charge density wave order in $\mathrm{YBa}_{2} \mathrm{Cu}_{3} \mathrm{O}_{6.67}$, Nat. Phys. 8, 871 (2012).

[10] D. LeBoeuf et al., Thermodynamic phase diagram of static charge order in underdoped $\mathrm{YBa}_{2} \mathrm{Cu}_{3} \mathrm{O}_{y}$, Nat. Phys. 9, 79 (2013).

[11] M. Hücker et al., Competing charge, spin, and superconducting orders in underdoped $\mathrm{YBa}_{2} \mathrm{Cu}_{3} \mathrm{O}_{y}$, Phys. Rev. B 90, 054514 (2014).

[12] M. Hashimoto et al., Direct observation of bulk charge modulations in optimally doped $\mathrm{Bi}_{1.5} \mathrm{~Pb}_{0.6} \mathrm{Sr}_{1.54} \mathrm{CaCu}_{2} \mathrm{O}_{8+\delta}$, Phys. Rev. B 89, 220511(R) (2014).
[13] S. Gerber et al., Three-dimensional charge density wave order in $\mathrm{YBa}_{2} \mathrm{Cu}_{3} \mathrm{O}_{6.67}$ at high magnetic fields, Science 350, 949 (2015).

[14] J. Chang et al., Magnetic field controlled charge density wave coupling in underdoped $\mathrm{YBa}_{2} \mathrm{Cu}_{3} \mathrm{O}_{6+x}$, Nat. Commun. 7, 11494 (2016).

[15] O. Cyr-Choinière et al., Sensitivity of $\mathrm{T}_{c}$ to pressure and magnetic field in the cuprate superconductor $\mathrm{YBa}_{2} \mathrm{Cu}_{3} \mathrm{O}_{y}$ : Evidence of charge order suppression by pressure, Phys. Rev. B 98, 064513 (2018).

[16] U. Ralević et al., Charge density wave modulation and gap measurements in $\mathrm{CeTe}_{3}$, Phys. Rev. B 94, 165132 (2016).

[17] E. H. da Silva Neto et al., Ubiquitous interplay between charge ordering and high-temperature superconductivity in cuprates, Science 343, 393 (2014).

[18] B. Loret, N. Auvray, Y. Gallais, M. Cazayous, A. Forget, D. Colson, M.-H. Julien, I. Paul, M. Civelli, and A. Sacuto, Intimate link between charge density wave, pseudogap and superconducting energy scales in cuprates, Nat. Phys. 15, 771 (2019).

[19] B. Keimer, S. A. Kivelson, M. R. Norman, S. Uchida, J. Zaanen, From quantum matter to high-temperature superconductivity in copper oxides, Nature (London) 518, 179 (2015).

[20] R. Arpaia et al., Dynamical charge density fluctuations pervading the phase diagram of a $\mathrm{Cu}$-based high- $T_{c}$ superconductor, Science 365, 906 (2019).

[21] Y. Y. Peng et al., Re-entrant charge order in overdoped $(\mathrm{Bi}, \mathrm{Pb})_{2.12} \mathrm{Sr}_{1.88} \mathrm{CuO}_{6+\delta}$ outside the pseudogap regime, Nat. Mater. 17, 697 (2018).

[22] E. Fradkin, S. A. Kivelson, and J. M. Tranquada, Colloquium: Theory of intertwined orders in high temperature superconductors, Rev. Mod. Phys. 87, 457 (2015).

[23] L. V. Delacrétaz, B. Goutéraux, S. A. Hartnoll, and A. Karlsson, Bad metals from fluctuating density waves, SciPost Phys. 3, 025 (2017). 
[24] L. V. Delacrétaz, and B. Goutéraux, A. A. Hartnoll, and A. Karlsson, Theory of hydrodynamic transport in fluctuating electronic charge density wave states, Phys. Rev. B 96, 195128 (2017).

[25] L. V. Delacrétaz, B. Goutéraux, S. A. Hartnoll, and A. Karlsson, Theory of the collective magnetophonon resonance and melting of the field-induced Wigner solid, Phys. Rev. B 100, 085140 (2019).

[26] A. Lucas, J. Crossno, K. C. Fong, P. Kim, and S. Sachdev, Transport in inhomogeneous quantum critical fluids and in the Dirac fluid in graphene, Phys. Rev. B 93, 075426 (2016).

[27] D. Di Sante et al., Turbulent hydrodynamics in strongly correlated Kagome metals, arXiv:1911.06810.

[28] A. A. Tsvetkov, J. Schützmann, J. I. Gorina, G. A. Kaljushnaia, and $\mathrm{D}$. van der Marel, In-plane optical response of $\mathrm{Bi}_{2} \mathrm{Sr}_{2} \mathrm{CuO}_{6}$, Phys. Rev. B 55, 14152 (1997).

[29] J. Hwang, T. Timusk, and G. D. Gu, Doping dependent optical properties of $\mathrm{Bi}_{2} \mathrm{Sr}_{2} \mathrm{CaCu}_{2} \mathrm{O}_{8+\delta}$, J. Phys.: Condens. Matter 19, 125208 (2007).

[30] O. Cyr-Choinière et al., Enhancement of the Nernst effect by stripe order in a high- $T_{c}$ superconductor, Nature (London) $\mathbf{4 5 8}$, 743 (2009).

[31] See Supplemental Material at http://link.aps.org/supplemental/ 10.1103/PhysRevResearch.2.023387 for details on the theoretical model and the experiment.

[32] H. Fukuyama and P. A. Lee, Dynamics of the charge-density wave. I. Impurity pinning in a single chain, Phys. Rev. B 17, 535 (1978)

[33] H. Fukuyama and P. A. Lee, Pinning and conductivity of twodimensional charge-density waves in magnetic fields, Phys. Rev. B 18, 6245 (1978).

[34] P. A. Lee and T. M. Rice, Electric field depinning of charge density waves, Phys. Rev. B 19, 3970 (1979).

[35] G. Grüner, The dynamics of charge-density waves, Rev. Mod. Phys. 60, 1129 (1988).

[36] A. Amoretti, D. Areán, B. Goutéraux, and D. Musso, A Holographic Strange Metal with Slowly Fluctuating Translational Order, Phys. Rev. Lett. 123, 211602 (2019).

[37] A. Amoretti, D. Areán, B. Goutéraux, and D. Musso, Diffusion and universal relaxation of holographic phonons, J. High Energ. Phys. 10 (2019) 068.

[38] R. M. Fleming et al., Stoichiometry and superconductivity in single layer $\mathrm{Bi}_{2+x} \mathrm{Sr}_{2-y} \mathrm{CuO}_{6 \pm \delta}$, Phys. C (Amsterdam, Neth.) 173, 37 (1991).
[39] N. R. Khasanova and E. V. Antipov, Bi-2201 phases synthesis, structures and superconducting properties, Phys. C (Amsterdam, Neth.) 246, 241 (1995).

[40] A. Piriou, E. Giannini, Y. Fasano, C. Senatore, and $\varnothing$ Fischer, Vortex phase diagram and temperature-dependent second-peak effect in overdoped $\mathrm{Bi}_{2} \mathrm{Sr}_{2} \mathrm{CuO}_{6+\delta}$ crystals, Phys. Rev. B 81, 144517 (2010).

[41] P. D. C. King et al., Structural Origin of Apparent Fermi Surface Pockets in Angle-Resolved Photoemission of $\mathrm{Bi}_{2} \mathrm{Sr}_{2-x} \mathrm{La}_{x} \mathrm{CuO}_{6+\delta}$, Phys. Rev. Lett. 106, 127005 (2011).

[42] S. Martin, A. T. Fiory, R. M. Fleming, L. F. Schneemeyer, and J. V. Waszczak, Normal-state transport properties of $\mathrm{Bi}_{2+x} \mathrm{Sr}_{2-y} \mathrm{CuO}_{6+\delta}$ crystals, Phys. Rev. B 41, 846 (1990).

[43] Y. Wang, L. Li, and N. P. Ong, Nernst effect in high- $T_{c}$ superconductors, Phys. Rev. B 73, 024510 (2006).

[44] C. Hess et al., Nernst effect of stripe ordering $\mathrm{La}_{1.8-x} \mathrm{Eu}_{0.2} \mathrm{Sr}_{x} \mathrm{CuO}_{4}$, Eur. Phys. J. Spec. Top. 188, 103 (2010).

[45] M. Blake and A. Donos, Quantum Critical Transport and the Hall Angle, Phys. Rev. Lett. 114, 021601 (2015).

[46] A. Amoretti, A. Braggio, N. Maggiore, and N. Magnoli, Adv. Phys. X 2, 409 (2017).

[47] A. Amoretti, A. Blasi, G. Caruso, N. Maggiore, and N. Magnoli, Eur. Phys. J. C 73, 2461 (2013).

[48] J. W. Loram, K. A. Mirza, J. R. Cooper, and W. Y. Liang, Electronic Specific Heat of $\mathrm{YBa}_{2} \mathrm{Cu}_{3} \mathrm{O}_{6+x}$ from 1.8 to $300 \mathrm{~K}$, Phys. Rev. Lett. 71, 1740 (1993).

[49] Y. Ando and T. Murayama, Nonuniversal power law of the Hall scattering rate in a single-layer cuprate $\mathrm{Bi}_{2} \mathrm{Sr}_{2-x} \mathrm{La}_{x} \mathrm{CuO}_{6}$, Phys. Rev. B 60, R6991 (1999).

[50] P. Coleman, A. J. Schofield, and A. M. Tsvelik, Phenomenological Transport Equation for the Cuprate Metals, Phys. Rev. Lett. 76, 1324 (1996).

[51] G. Grissonnanche et al., Giant thermal Hall conductivity in the pseudogap phase of cuprate superconductors, Nature (London) 571, 376 (2019).

[52] Y. Zhang, N. P. Ong, and Z. A. Xu, K. Krishana, R. Gagnon, and L. Taillefer, Determining the Wiedemann-Franz Ratio from the Thermal Hall Conductivity: Application to $\mathrm{Cu}$ and $\mathrm{YBa}_{2} \mathrm{Cu}_{3} \mathrm{O}_{6.95}$, Phys. Rev. Lett. 84, 2219 (2000).

[53] M. Matusiak, K. Rogacki, and B. W. Veal, Enhancement of the Hall-Lorenz number in optimally doped $\mathrm{YBa}_{2} \mathrm{Cu}_{3} \mathrm{O}_{7-d}$, Europhys. Lett. 88, 47005 (2009). 